\title{
Pathways for the Spread of Disease in the Abdomen and Pelvis
}

\author{
James A. Brink and Brent J. Wagner
}

\section{Learning Objectives}

- To discuss the ligamentous anatomy of the upper abdomen and how it can inform the spread of disease by direct invasion and lymphatic extension

- To learn about the anatomy of the peritoneal spaces and how it can inform the spread of disease by intraperitoneal seeding

\subsection{Introduction}

Disease may spread through the abdomen and pelvis by a variety of mechanisms. For example, intra-abdominal malignancies may metastasize through hematologic routes, and tumors may spread by directly invading adjacent tissues and organs or via the lymphatic system. When tumors break through the visceral peritoneum, they may also spread via intraperitoneal seeding. While hematologic spread of disease is beyond the scope of this chapter, direct invasion, lymphatic extension, and intraperitoneal seeding will be discussed relative to the anatomy that guides these pathways for the spread of disease in the abdomen and pelvis [1].

Direct invasion and lymphatic extension occur through the peritoneal ligaments and mesenteries that interconnect the abdominal viscera with other organs in the abdomen and pelvis, the retroperitoneum, and the body wall. Moreover, these structures guide the flow of peritoneal fluid through the abdomen and pelvis, thereby dictating the routes of spread through intraperitoneal seeding. In short, understanding these pathways

\footnotetext{
J. A. Brink, M.D. $(\bowtie)$

Department of Radiology, Massachusetts General Hospital, Boston, MA, USA

e-mail: jabrink@partners.org

B. J. Wagner, M.D.

West Reading Radiology Associates, West Reading, PA, USA

e-mail: Brent.Wagner@ towerhealth.org
}

for the spread of disease ties closely to a clear understanding of a ligamentous anatomy of the abdomen and pelvis.

\subsection{Peritoneal Ligaments as Conduits for the Spread of Disease}

The upper abdominal viscera is interconnected by three pairs of ligaments: the gastrohepatic and hepatoduodenal ligaments (that together comprise the lesser omentum), the gastrosplenic and splenorenal ligaments, and the gastrocolic ligament and transverse mesocolon. Each of these ligamentous pairs contains one ligament that bridges to the retroperitoneum: the hepatoduodenal ligament, the splenorenal ligament, and the transverse mesocolon. Thus, disease from the abdominal viscera may spread to the retroperitoneum and vice versa through these ligamentous pairs.

\subsubsection{Gastrohepatic and Hepatoduodenal Ligaments}

The gastrohepatic and hepatoduodenal ligaments form an important pathway of disease from the lesser curvature of the stomach to the porta hepatis and retroperitoneum. The gastrohepatic ligament extends from the lesser curvature of the stomach to the porta hepatis, inserting into the fissure for the ligamentum venosum. Containing the left gastric artery, the left gastric vein or coronary vein, and associated lymphatics, the gastrohepatic ligament may be recognized on crosssectional imaging as the fatty plane connecting the lesser curvature of the stomach to the left lobe of the liver and containing these vessels (Fig. 6.1). Nodes in the gastrohepatic ligament are typically $8 \mathrm{~mm}$ or less in diameter, somewhat smaller than elsewhere in the abdomen [2]. Care must be taken to avoid misidentifying unopacified loops of bowel, the pancreatic neck, or the papillary process of the caudate lobe as enlarged nodes in the gastrohepatic ligament $[3,4]$. 


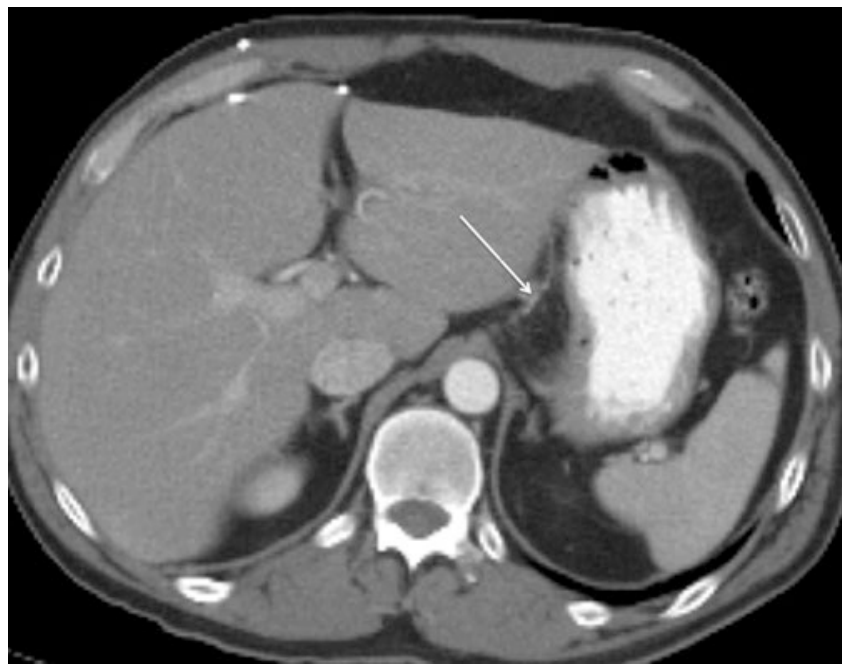

Fig. 6.1 Transaxial CT image demonstrates the gastrohepatic ligament (GHL), seen as a fatty plane interposed between the lesser curvature of the stomach and the lobe of the liver. The GHL contains the left gastric artery (arrow), the left gastric vein (coronary vein), and associated lymphatics

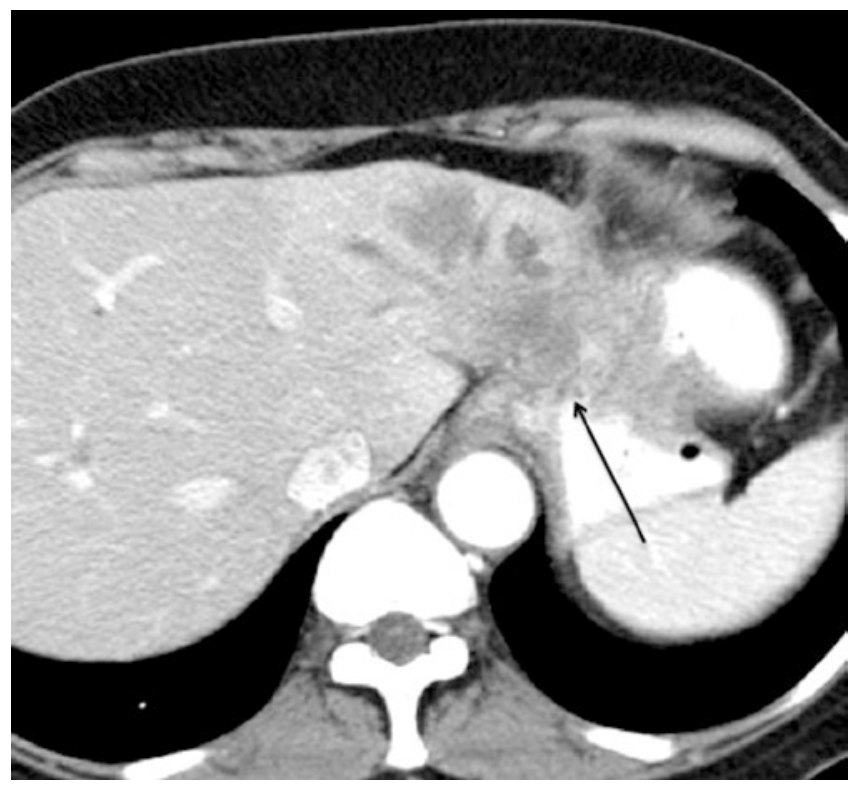

Fig. 6.2 Transaxial CT image demonstrates a heterogenous mass (arrow) centered in the gastrohepatic ligament (GHL), with invasion of the left hepatic lobe and the stomach. Prospectively, it was unclear as to the organ of origin, but it proved to be a hepatoma extending through the GHL to involve the stomach

A unique feature of the gastrohepatic ligament is continuity of its subperitoneal areolar tissue with the perivascular fibrous capsule of the liver (Glisson capsule). This anatomic continuity provides a direct pathway for the spread of disease from the gastric lesser curvature into the left hepatic lobe via the gastrohepatic ligament and vice versa. Both neoplastic and inflammatory conditions can spread in this fashion (Fig. 6.2). Gastric and esophageal cancer commonly spread via lymphatic extension and direct invasion through the gastrohepatic ligament, allowing these tumors to spread to the liver, and conversely hepatoma and cholangiocarcinoma may spread to the stomach through these pathways as well.

The free edge of the gastrohepatic ligament is the hepatoduodenal ligament, and together, the gastrohepatic and hepatoduodenal ligaments comprise the lesser omentum. The hepatoduodenal ligament is the thickest ligament in the upper abdomen owing to the portal structures that it contains: the portal vein, the hepatic artery, the common bile duct, and associated lymphatics. The hepatoduodenal ligament extends from the porta hepatis to the flexure between the first and second duodenum forming a tent-like structure that extends from superior to inferior as it courses from anterior to posterior. The foramen of Winslow, or epiploic foramen, lies immediately posterior to the ligament connecting the right posterior perihepatic space with the lesser sac [5]. Here, nodes at the base of the hepatoduodenal ligament at the epiploic foramen (in the portocaval space) can be quite prominent in size and still normal. These nodes can be up to $2.0 \mathrm{~cm}$ in transverse dimension and up to $1.5 \mathrm{~cm}$ in anteroposterior dimension and still be normal. Pathology within these nodes may be difficult to identify but may be suggested when the nodes assume a more spherical shape or have central necrosis $[6,7]$.

A host of neoplastic and inflammatory conditions spread commonly via the hepatoduodenal ligament from the porta hepatis to the retroperitoneum, following antegrade flow of lymphatic fluid from the liver and biliary tree to nodes surrounding the duodenum and pancreas. However, lymphatic extension can also occur in a retrograde fashion through these lymphatics, originating from disease in nodes surrounding the superior mesenteric artery, as may occur in pancreatic and colon cancer, and spreading up the lymphatic channels in the hepatoduodenal ligament to the liver (Fig. 6.3).

Structures intimately related to the hepatoduodenal ligament may also spread via direct invasion through the ligament, as occurs commonly with gastric cancer arising in the lesser curvature and spreading to peripancreatic and periduodenal lymph nodes via the gastrohepatic and hepatoduodenal ligaments. Many inflammatory conditions also spread commonly through the hepatoduodenal ligament including inflammatory processes within the gall bladder and biliary tree. Pancreatitis may also spread via this pathway as well. Occasionally, vascular complications may be seen in the hepatoduodenal ligament related to both malignant and inflammatory conditions coursing through it. These include portal vein thrombosis as well as hepatic arterial pseudoaneurysms $[1,5]$.

\subsubsection{Gastrosplenic and Splenorenal Ligaments}

An important highway of disease is provided in the left upper abdomen by the gastrosplenic and splenorenal ligaments, connecting the gastric greater curvature to the splenic hilum and the retroperitoneum, respectively (Fig. 6.4). The gastrosplenic 


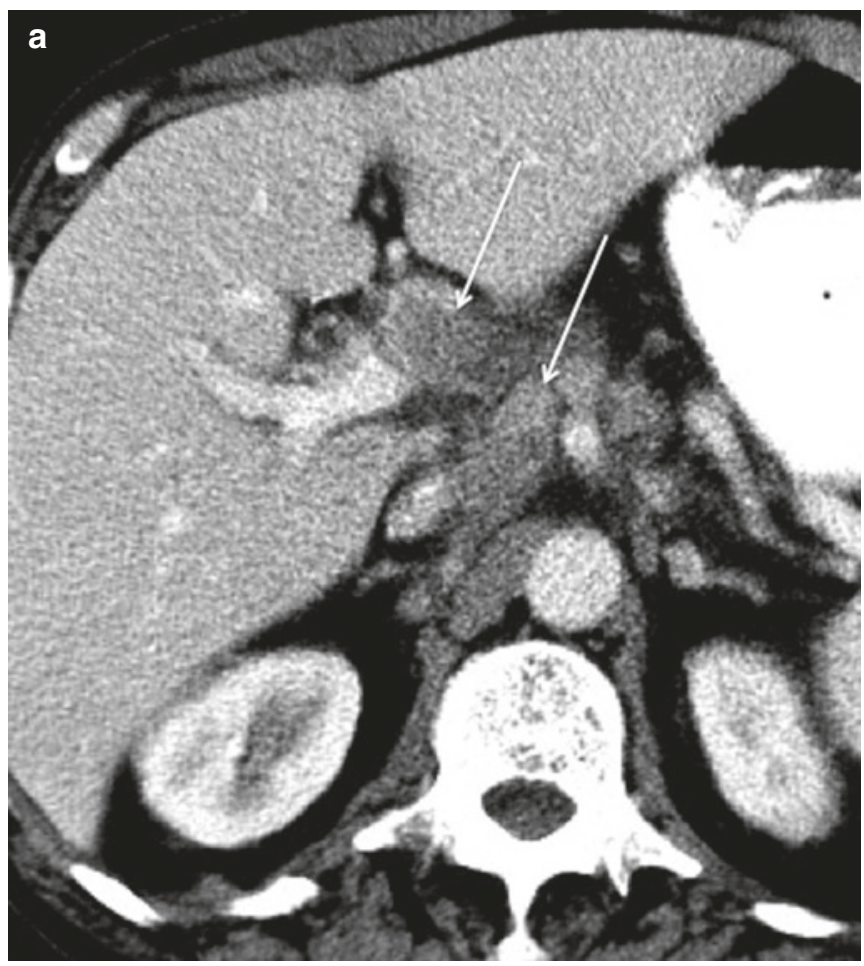

Fig. 6.3 Transaxial CT images through the porta hepatis (a) and the uncinate process (b) demonstrate bulky lymphadenopathy (arrows) in the hepatoduodenal ligament (HDL) in a patient with gallbladder carci-

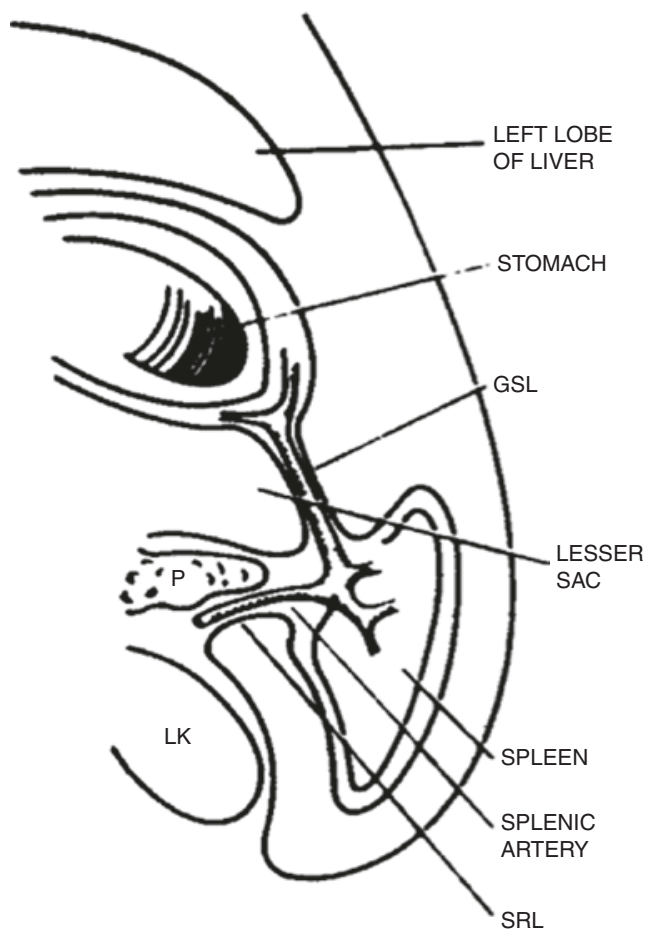

Fig. 6.4 The gastrosplenic ligament (GSL) and splenorenal ligament (SRL) comprise the left wall of the lesser sac and provide a conduit for the spread of metastatic disease from the greater curvature of the stomach to the retroperitoneum and vice versa (Reprinted with permission from Myers MA. Dynamic Radiology of the Abdomen: Normal and Pathologic Anatomy, New York: Springer, 1994)

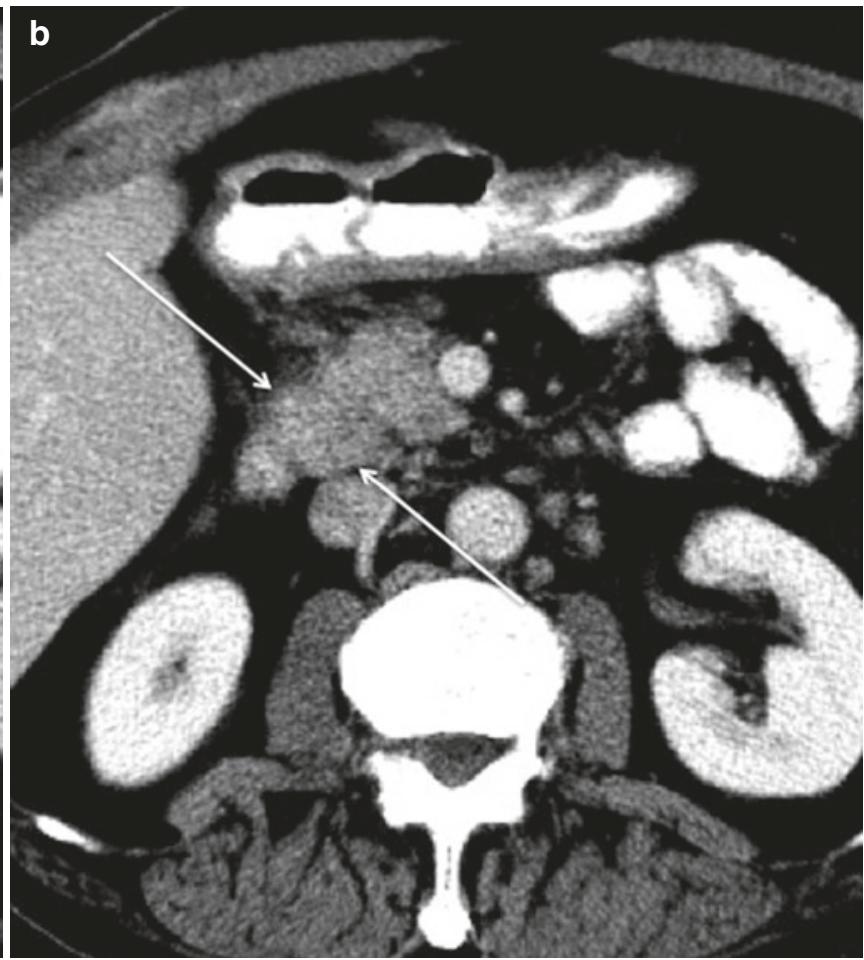

noma. Tumor has spread bidirectionally within the HDL proximally (a) and distally to the insertion of the HDL on the second portion of the duodenum

ligament is a rather thin delicate structure that connects the superior third of the greater curvature of the stomach to the splenic hilum. This ligament contains the left gastroepiploic and short gastric vessels and their associated lymphatics. The gastrosplenic ligament can direct diseases arising in the stomach to the splenic hilum, and both neoplastic and inflammatory disease may invade the spleen via this pathway.

Posteriorly and medially, the gastrosplenic ligament is continuous with the splenorenal ligament. Once disease reaches the splenic hilum from the stomach via the gastrosplenic ligament, it may turn and extend to the retroperitoneum via the splenorenal ligament. Here, disease may surround and invade the pancreatic tail and compromise the splenic artery and splenic vein (Fig. 6.5) [8, 9]. Just as gastric disease can spread to the retroperitoneum via this ligamentous pair, both inflammatory and neoplastic disease of the pancreas may spread in the opposite direction to the splenic hilum and greater curvature via the splenorenal and gastrosplenic ligaments, respectively [1].

\subsubsection{Gastrocolic Ligament and Transverse Mesocolon}

As the gastrohepatic and hepatoduodenal ligaments in the right abdomen and the gastrosplenic and splenorenal ligaments in the left abdomen form important pathways of 

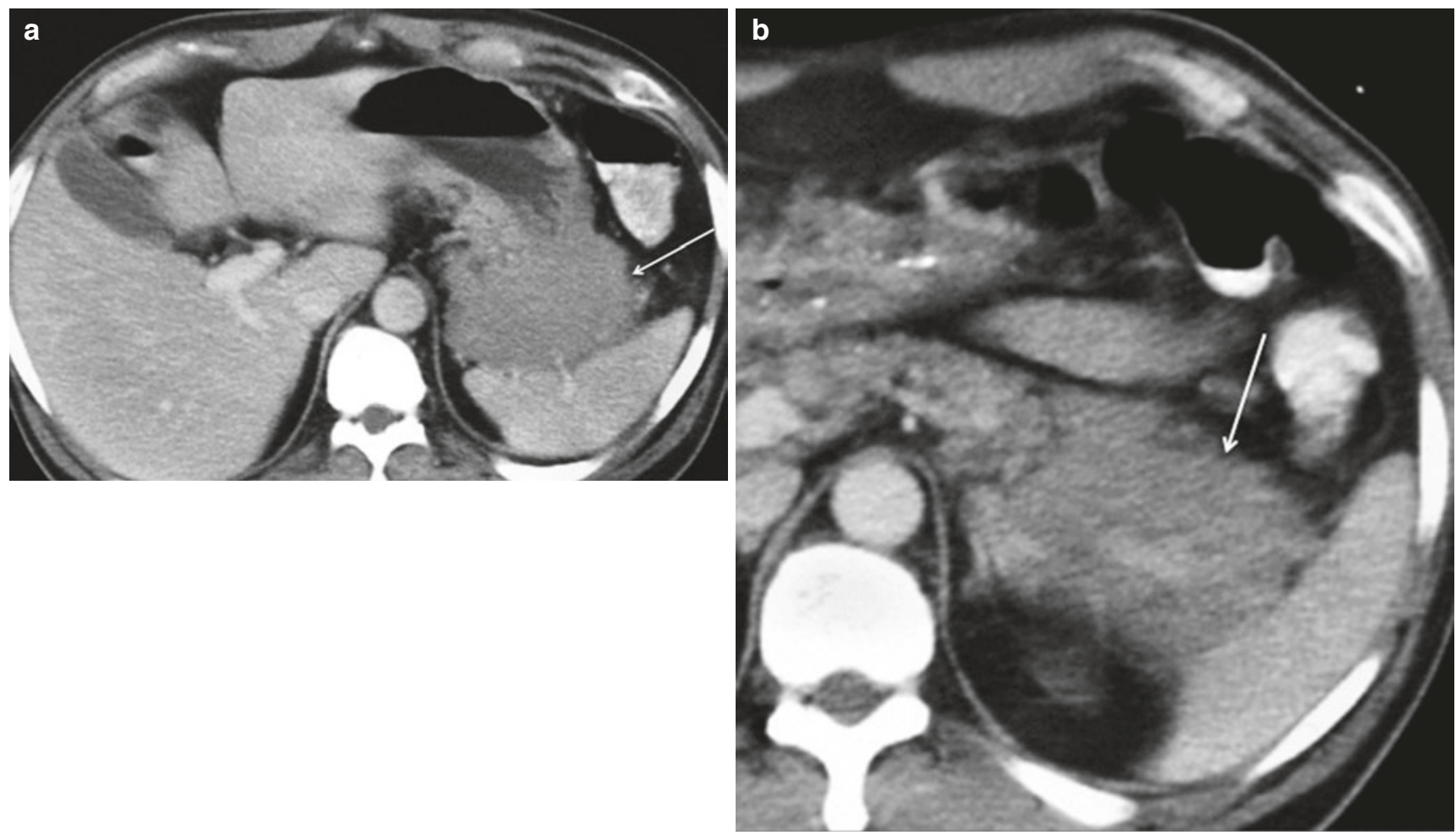

Fig. 6.5 Transaxial CT images through the gastrosplenic ligament (GSL) (a) and the splenorenal ligament (SRL) (b), in a patient with lymphoma. Tumor is seen within the GSL, interposed between the gas-

disease from the upper abdominal viscera to the retroperitoneum, the gastrocolic ligament and transverse mesocolon form a similar pathway in the mid-abdomen. The gastrocolic ligament (greater omentum) connects the inferior two thirds of the greater curvature of the stomach to the transverse colon (Fig. 6.6). On the left, the gastrocolic ligament is continuous with the gastrosplenic ligament, and on the right, it ends at the gastroduodenal junction near the hepatoduodenal ligament. Embryologically, the gastrosplenic ligament gives rise to the gastrocolic ligament and the transverse mesocolon in the adult, with fusion of the anterior and posterior leaves of the embryonic gastrosplenic ligament. In consequence, the gastrocolic ligament has a potential space within it that can fill with fluid when tense ascites in the lesser sac dissects open this potential space. This can result in a cyst-like appearance within the gastrocolic ligament/greater omentum.

The gastrocolic ligament contains the gastroepiploic vessels and associated lymphatics which can help identify the ligament as the fatty plane connecting the stomach to the transverse colon. Both benign and malignant diseases from the inferior two thirds of the greater curvature of the stomach may spread to the transverse colon via this pathway, and vice versa (Fig. 6.7). The transverse mesocolon completes the pathway from the stomach to the retroperitoneum in the midabdomen; disease involving the stomach and transverse colon are connected via the gastrocolic ligament, and disease involving the transverse colon and pancreas/retroperitoneum

tric greater curvature and the spleen (a), and within the SRL, encasing the splenic vasculature (b)

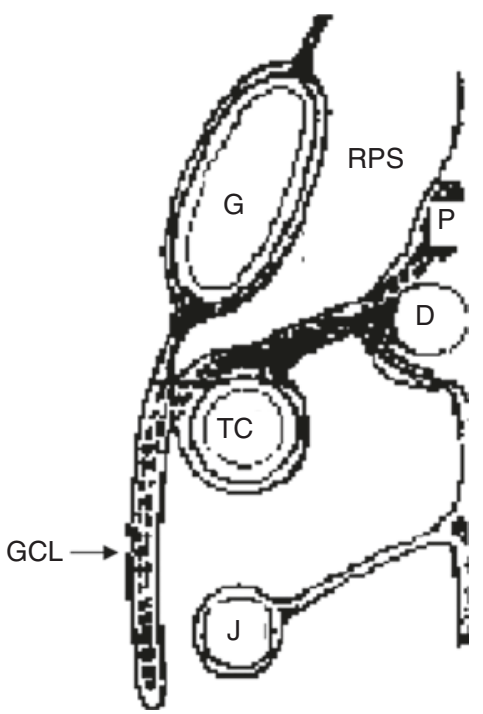

Fig. 6.6 The gastrocolic ligament (GCL) joins the greater curvature of the stomach $(\mathrm{G})$ to the transverse colon (TC). In concert with the transverse mesocolon, a pathway of disease is formed between retroperitoneal structures such as the pancreas (P) and the duodenum (D) to the anterior aspect of the intraperitoneal cavity (Modified from Langman J., Medical Embriology, New York: Saunders, 1971)

are connected by the transverse mesocolon. In addition, the greater omentum continues inferior to the transverse colon as a fatty veil that forms an important nidus for carcinomatosis, as commonly occurs with ovarian, gastric, colon, and pancre- 


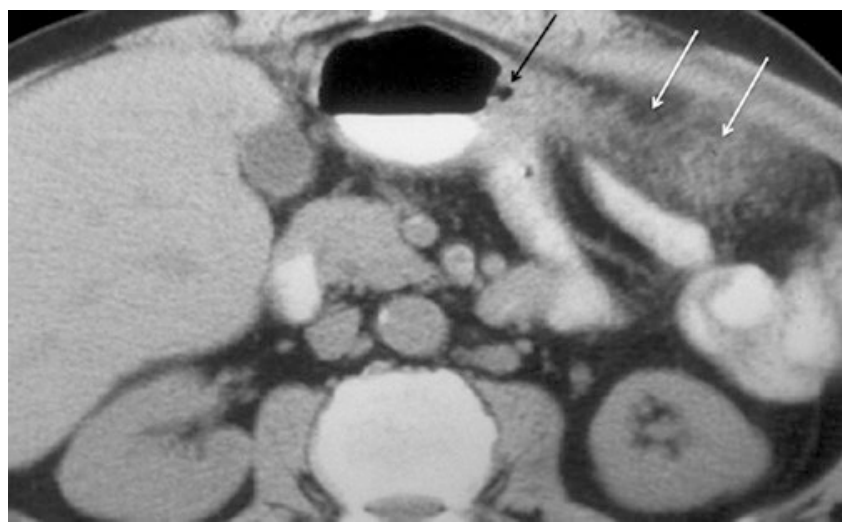

Fig. 6.7 Transaxial CT image through the gastrocolic ligament (GCL) demonstrates a gastric ulcer extending into the GCL (black arrow) with associated inflammation in the greater omentum (white arrows)

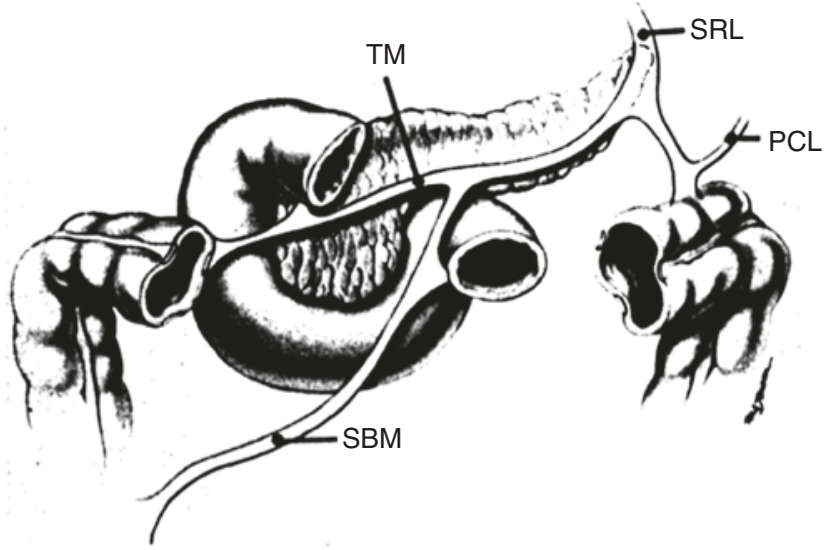

Fig. 6.8 The transverse mesocolon (TM) provides an important conduit for the spread of disease across the mid-abdomen. It is continuous with the splenorenal ligament (SRL) and phrenicocolic ligament (PCL) on the left and with the duodenocolic ligament on the right. In its midportion, it is continuous with the small bowel mesentery (SBM) (Reprinted with permission from Myers MA. Dynamic Radiology of the Abdomen: Normal and Pathologic Anatomy, New York: Springer, 1994)

atic cancers $[10,11]$. Sometimes, gastroepiploic collaterals may be recognized in the gastrocolic ligament which should raise concern about the possibility of splenic venous compromise as what commonly occurs in pancreatic carcinoma.

The transverse mesocolon connects the transverse colon to the retroperitoneum but also forms a broad conduit for disease across the mid-abdomen; bare areas link the pancreas to the transverse colon, spleen, and small bowel (Fig. 6.8). The transverse mesocolon is continuous with the phrenicocolic ligament and the splenorenal ligaments in the left abdomen, with the small bowel mesentery in the mid-abdomen, and with the duodenocolic ligament in the right abdomen. The transverse mesocolon may be recognized as the fatty plane that connects the transverse colon to the retroperitoneum at the level of the uncinate process of the pancreas. As this structure lies commonly in a paracoronal orientation, recog-

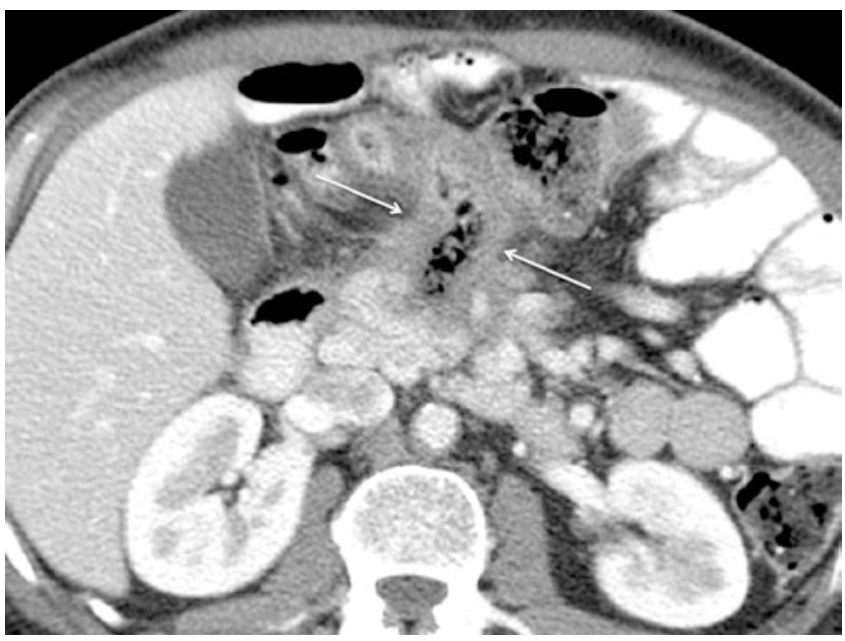

Fig. 6.9 Transaxial CT image through the transverse mesocolon (TMC) in a patient with pancreatic adenocarcinoma demonstrates invasion of the TMC with necrotic tumor (arrows). The tumor in the TMC has fistulized with the transverse colon resulting in gas accumulating within the necrotic debris

nition of the middle colic vessels and associated lymphatics within the mesocolon can aid in its identification. Pancreatic disease, both benign and malignant, often spreads ventrally into the transverse mesocolon and then on to the transverse colon (Fig. 6.9). Pancreatitis often results in adjacent fluid collections that can dissect open the potential space within the transverse mesocolon formed by fusion of the anterior and posterior leaves of the embryonic gastrosplenic ligament. Free fluid in the lesser sac is often confused with contained fluid collections within the transverse mesocolon.

The duodenocolic ligament, the right edge of the transverse mesocolon, forms an important pathway for the spread of right colon cancers via lymphatic drainage from the right colon passing through this ligament. Tumors of the right colon may spread via these lymphatics to deposit in nodes around the duodenum and pancreas [1]. Gastric outlet obstruction may occur once this adenopathy becomes sufficiently severe to obstruct the second duodenum explaining how cancers of the right colon may result in upper gastrointestinal obstruction on rare occasion.

\subsection{Peritoneal Spaces as Pathways for the Spread of Disease}

Peritoneal fluid flows naturally through the peritoneal spaces that are defined by the peritoneal ligaments and mesenteries in the abdomen and pelvis. However, certain neoplastic and inflammatory conditions within the peritoneal cavity may leverage this natural flow of peritoneal fluid to spread throughout the peritoneal spaces. Tumors that arise from the peritoneal lining or break through the visceral peritoneum may shed their cells directly into the peritoneal fluid. Similarly, 
inflammatory processes within the peritoneal cavity may also leverage this natural flow of ascitic fluid and spread throughout the peritoneal spaces of the abdomen and pelvis. A thorough knowledge and understanding of this anatomy can help narrow the differential diagnosis for intra-abdominal pathologies and may help radiologists better predict the organ of origin and likely route of spread for certain conditions (Fig. 6.10).

\subsubsection{Left Peritoneal Space}

The left peritoneal space is comprised of four compartments: the left anterior perihepatic space, the left posterior perihepatic space (gastrohepatic recess), the left anterior subphrenic space, and the left posterior subphrenic space (perisplenic space).

Anteriorly, the left peritoneal space extends to the right as the left anterior perihepatic space, ventral to the left lobe of the liver. It is bounded laterally on the right by the falciform ligament and on the left by the anterior wall of the stomach. Posteriorly, it extends along the diaphragm and is limited by the left coronary ligament, the left superior extension of the bare area of the liver (Fig. 6.11a).

Along the medial margin of the left hepatic lobe, the left anterior perihepatic space turns to form the left posterior perihepatic space as it extends along the inferior margin of the left hepatic lobe posteriorly, deep into the fissure for the

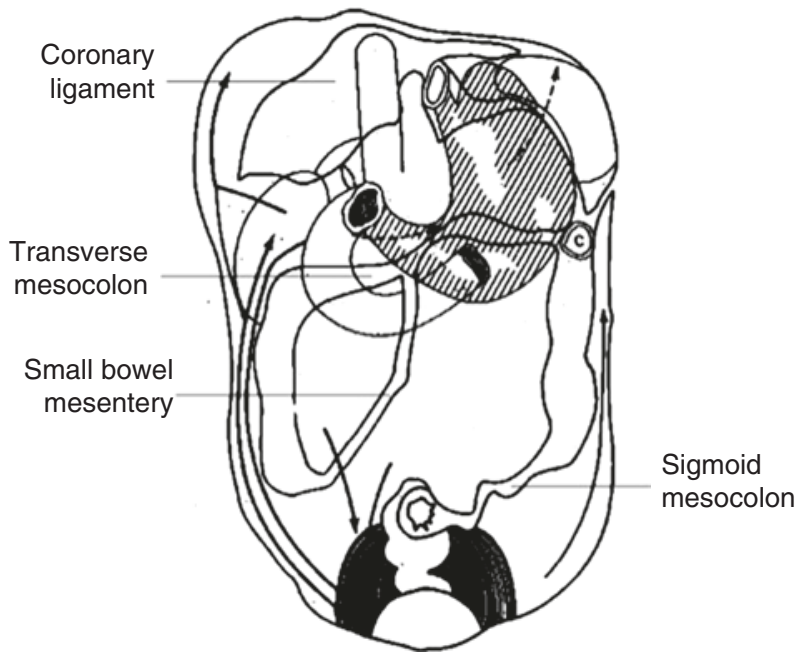

Fig. 6.10 Posterior peritoneal reflections and recesses. Intraperitoneal fluid flows naturally from the pelvis to the upper abdomen. Flow occurs preferentially through the right rather than left paracolic gutters owing to the broader diameter of the right gutter. In addition, flow in the left paracolic gutter is cut off from reaching the left subphrenic space by the phrenicocolic ligament. The transverse mesocolon divides the abdomen into supra- and inframesocolic spaces. In the right inframesocolic space, fluid is impeded from draining into the pelvis via the small bowel mesentery. Owing to natural holdup of fluid at the root of the small bowel mesentery and sigmoid mesocolon, these structures are naturally predisposed to involvement with serosal-based metastases in the setting of peritoneal carcinomatosis (Reprinted with permission from Myers MA. Dynamic Radiology of the Abdomen: Normal and Pathologic Anatomy, New York: Springer, 1994)

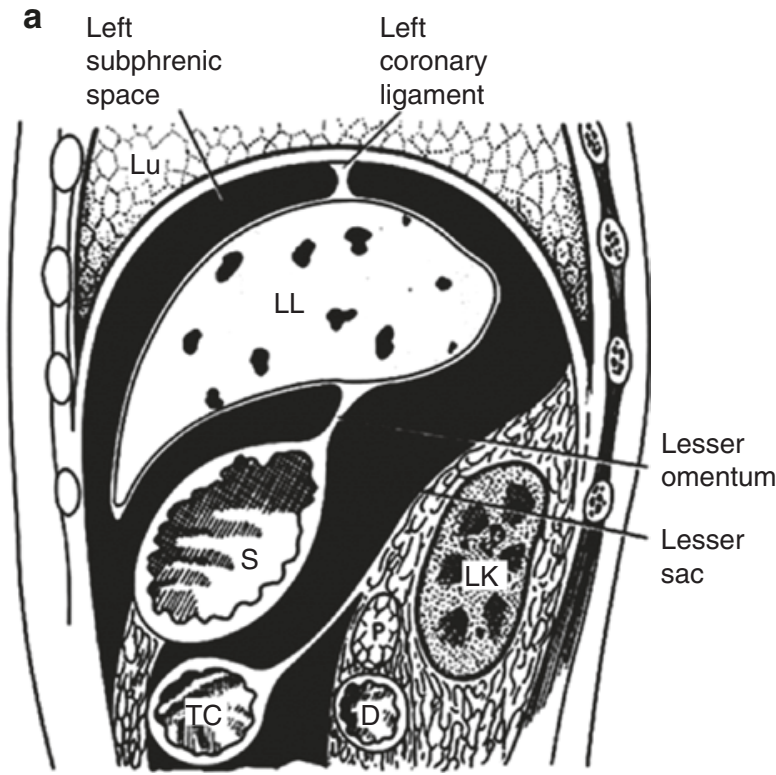

Fig. 6.11 Left (a) and right (b) perihepatic spaces. The left and right perihepatic spaces are bounded posteriorly by the coronary ligaments. The reflections of the coronary ligaments mark the site of the nonperitonealized "bare area" of the liver ( $L L$ left lobe of the liver, $L K$ left b

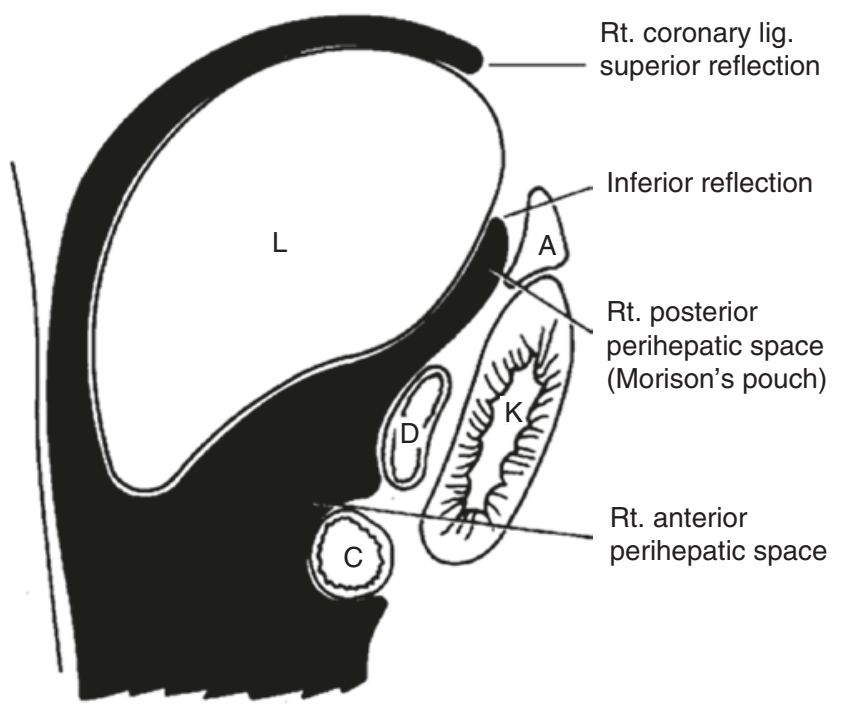

kidney, $S$ stomach, $T C$ transverse colon, $P$ pancreas, $D$ duodenum, $L u$ lung, $L$ liver [right lobe], $A$ adrenal, $K$ kidney, $C$ colon) (Reprinted with permission from Myers MA. Dynamic Radiology of the Abdomen: Normal and Pathologic Anatomy, New York: Springer, 1994) 
ligamentum venosum. Also known as the gastrohepatic recess, the left posterior perihepatic space is bounded on the left by the lateral wall of the stomach and is juxtaposed to the anterior wall of the duodenal bulb, the anterior wall of the gallbladder, and the porta hepatis [8]. Fluid in the gastrohepatic recess is separated from fluid in the superior recess of the lesser sac by the lesser omentum as it inserts into the fissure for the ligamentum venosum. Fluid collections in the gastrohepatic recess are relatively easy to drain owing to the lack of intervening structures between this space and the body wall, along the medial margin of the left hepatic lobe. Conversely, fluid collections in the lesser sac may be more difficult to approach percutaneously owing to the presence of intervening vasculature in the lesser omentum.

Laterally in the left abdomen, the left anterior subphrenic space connects with the left anterior perihepatic space across the mid-abdomen. This space is cut off from the left paracolic gutter by the phrenicocolic ligament, unlike the right subphrenic space which communicates freely with the right paracolic gutter. Thus, fluid can accumulate within the left anterior subphrenic space by passing ventral to the stomach, but once it enters this space, it is relatively static owing to the phrenicocolic ligament. Thus, fluid in the left anterior subphrenic space is a common site for peritoneal carcinomatosis and abscess formation consequent to peritonitis [12].

The posterior extension of the left anterior subphrenic space is the left posterior subphrenic space also known as the perisplenic space (Fig. 6.12). The bare areas of the spleen

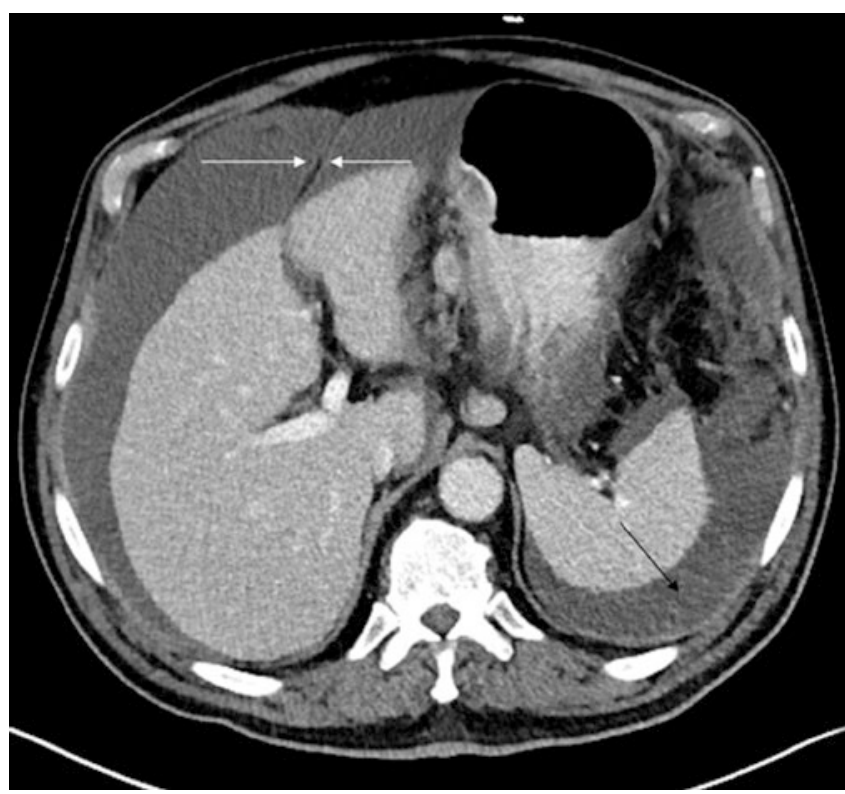

Fig.6.12 Transaxial CT image from a patient with carcinomatosis secondary to gastric cancer demonstrates fluid in the left posterior subphrenic space (black arrow) and fluid in the right and left anterior perihepatic spaces, separated by the falciform ligament (white arrows) that result from insertion of the gastrosplenic and splenorenal ligaments into the splenic hilum may be highlighted by fluid that surrounds the spleen within the perisplenic space [13-15]. Superiorly, the perisplenic space surrounds completely the upper margin of the spleen [16].

\subsubsection{Right Peritoneal Space}

The right peritoneal space is comprised of three compartments: the right subphrenic space/right anterior perihepatic space, the right posterior perihepatic space (hepatorenal recess/Morison's pouch), and the lesser sac.

The right subphrenic space surrounds the upper margin of the liver, separating it from the right hemidiaphragm (Figs. 6.11b and 6.12). Posteriorly and medially, the right coronary ligament (bare area of the liver) forms the posteromedial border of the right subphrenic space [17] (Fig. 6.13).

Inferior to the right coronary ligament, the hepatorenal recess (Morison's pouch) is the medial extension of the right subphrenic space, located between the right lobe of the liver and the anterior border of the kidney.

The lesser sac is the leftward extension of the right posterior perihepatic space/hepatorenal recess/Morison's pouch as it extends through the foramen of Winslow. The lesser sac is comprised of superior and inferior recesses $[9,18]$. Fluid in the superior recess of the lesser sac surrounds the caudate lobe producing a reverse $\mathrm{C}$-shaped configuration as it surrounds this structure (Figs. 6.14 and 6.15). A raised peritoneal reflection along the posterior aspect of the lesser sac serves as an anatomic boundary that separates the superior from the inferior recesses. This gastropancreatic plicae contains the proximal left gastric artery and may be recognized, particularly when surrounded by ascitic fluid in the superior and inferior recesses.

The inferior recess of the lesser sac is bounded laterally by the gastrosplenic and splenorenal ligaments, inferoposteriorly by the transverse mesocolon, and anteriorly by the stomach. Percutaneous drainage of fluid in lesser sac collections is problematic owing to the presence of abdominal organs, ligaments, and mesenteries that surround both superior and inferior recesses completely [19].

\subsection{Concluding Remarks}

Upper abdominal disease may spread from the upper abdominal organs to the retroperitoneum and vice versa via the gastrohepatic and hepatoduodenal ligaments in the right abdomen, the gastrosplenic and splenorenal ligaments in the left abdomen, and the gastrocolic ligament and transverse mesocolon in the mid-abdomen. Disease in any of these ligamentous pairs can suggest the organ of origin and, in 

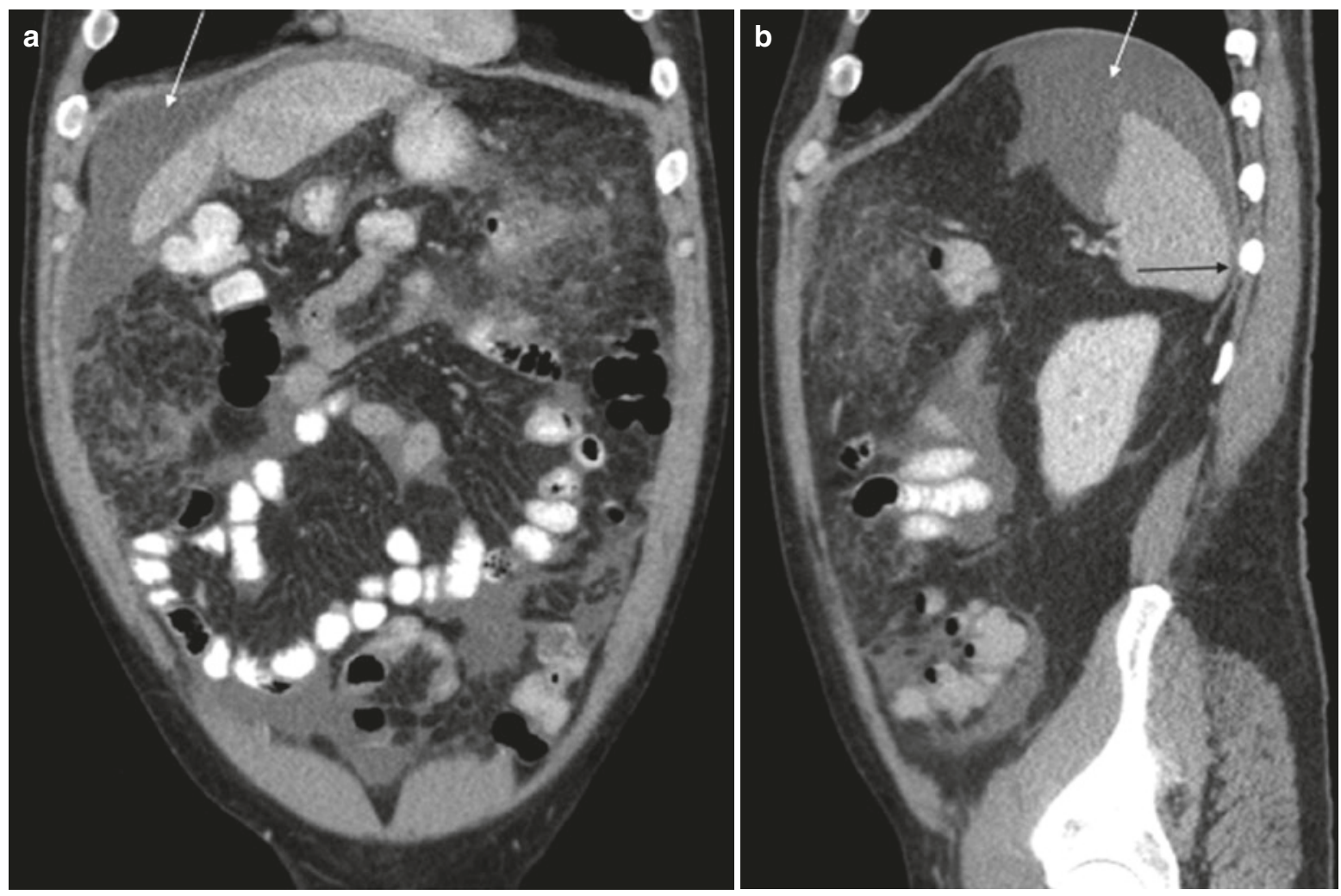

Fig. 6.13 Coronal (a) and sagittal (b) reformatted CT images from a patient with peritoneal mesothelioma demonstrate fluid in the right subphrenic space (white arrows) bounded posteriorly by the right coronary ligament (black arrow)

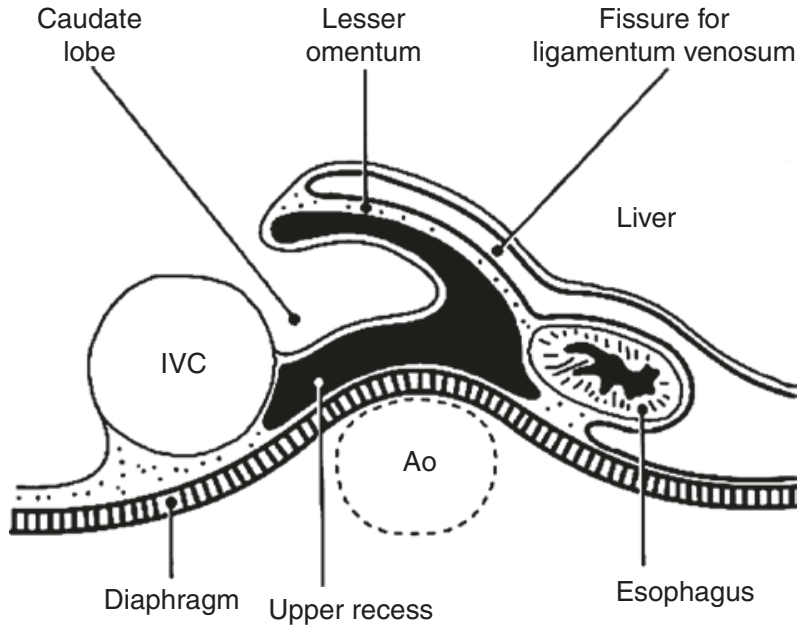

Fig. 6.14 The boundaries of the superior recess of the lesser sac may be recognized when fluid engulfs the caudate lobe. The lesser omentum separates this fluid from fluid in fissure for the ligamentum venosum which is in continuity with the left posterior perihepatic space (gastrohepatic recess) (IVC inferior vena cava, Ao aorta) (Reprinted with permission from Myers MA. Dynamic Radiology of the Abdomen: Normal and Pathologic Anatomy, New York: Springer, 1994)

some cases, the location of the disease within the organ. Peritoneal ligaments and mesenteries also guide the flow of intraperitoneal fluid throughout the peritoneal spaces that they define. Neoplastic and inflammatory diseases that arise

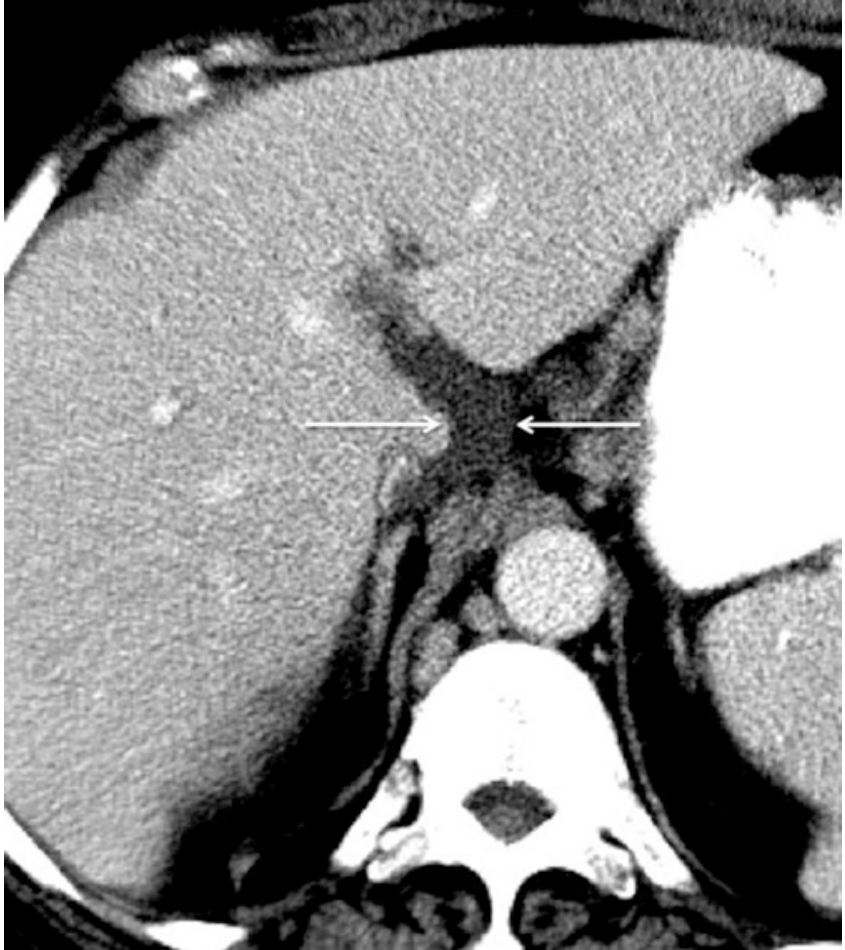

Fig. 6.15 Transaxial CT image through the superior recess of the lesser sac in a patient with gallbladder cancer (same patient as is illustrated in Fig. 6.3). Malignant ascites has accumulated in the superior recess of the lesser sac (arrows), seen with a characteristic reverse $\mathrm{C}$-shaped configuration surrounding the caudate lobe 
de novo or extend into the peritoneal cavity may spread through these spaces and deposit in predictable areas within the peritoneal cavity.

\section{Key Points}

- Disease may spread through the abdomen and pelvis by the following mechanisms:

via the bloodstream

via lymphatic extension

via direct invasion

via intraperitoneal seeding

- Direct invasion and lymphatic extension occur commonly through the peritoneal ligaments and mesenteries that interconnect the abdominal viscera

- Intraperitoneal spread occurs by seeding the peritoneal cavity with cells that spread via the natural flow of peritoneal fluid via the peritoneal spaces

\section{Take-Home Message}

A thorough understanding of the peritoneal ligaments and mesenteries as well as the peritoneal spaces that they define can inform the pathways by which inflammatory and neoplastic diseases may spread throughout the abdomen and pelvis.
3. Auh YH, Rosen A, Rubenstein WA, Engel IA, Whalen JP, Kazam E. CT of the papillary process of the caudate lobe of the liver. AJR. 1984;142:535-8.

4. Donoso L, Martinez-Noguera A, Zidan A, Lora F. Papillary process of the caudate lobe of the liver: sonographic appearance. Radiology. 1989;173:631-3.

5. Weinstein JB, Heiken JP, Lee JKT, DiSantis DJ, Balfe DM, Weyman PJ, Peterson RR. High resolution CT of the porta hepatis and hepatoduodenal ligament. Radiographics. 1986;6:55-74.

6. Zirinsky K, Auh YH, Rubenstein WA, Kneeland JB, Whalen JP, Kazam E. The portacaval space: CT with MR correlation. Radiology. 1985;156:453-60.

7. Ito K, Choji T, Fujita T, Kuramitsu T, Nakaki H, Kurokawa F, Fujita N, Nakanishi T. Imaging of the portacaval space. AJR. 1993;161:329-34.

8. Vincent LM, Mauro MA, Mittelstaedt CA. The lesser sac and gastrohepatic recess: sonographic appearance and differentiation of fluid collections. Radiology. 1984;150:515-9.

9. Dodds WJ, Foley WD, Lawson TL, Stewart ET, Taylor A. Anatomy and imaging of the lesser peritoneal sac. AJR. 1985;144: $567-75$.

10. Cooper C, Jeffrey RB, Silverman PM, Federle MP, Chun GH. Computed tomography of omental pathology. J Comput Assist Tomogr. 1986;10(1):62-6.

11. Rubesin SE, Levine MS, Glick SN. Gastric involvement by omental cakes: radiographic findings. Gastrointest Radiol. 1986;11:223-8.

12. Halvorsen RA, Jones MA, Rice RP, Thompson WM. Anterior left subphrenic abscess: characteristic plain film and CT appearance. AJR. 1982;139:283-9.

13. Vibhakar SD, Bellon EM. The bare area of the spleen: a constant CT feature of the ascitic abdomen. AJR. 1984;141:953-5.

14. Rubenstein WA, Auh YH, Zirinsky K, Kneeland JB, Whalen JP, Kazam E. Posterior peritoneal recesses: assessment using CT. Radiology. 1985;156:461-8.

15. Love L, Demos TC, Posniak H. CT of retrorenal fluid collections. AJR. 1985;145:87-91.

16. Crass JR, Maile CW, Frick MP. Catheter drainage of the left posterior subphrenic space: a reliable percutaneous approach. Gastrointest Radiol. 1985;10:397-8.

17. Rubenstein WA, Auh TH, Whalen JP, Kazem E. The perihepatic spaces: computed tomographic and ultrasound imaging. Radiology. 1983;149:231-9.

18. Jeffrey RB, Federle MP, Goodman PC. Computed tomography of the lesser peritoneal sac. Radiology. 1981;141:117-22.

19. Meyers MA. Dynamic radiology of the abdomen: normal and pathologic anatomy. 4th ed. New York: Springer; 1994.

Open Access This chapter is licensed under the terms of the Creative Commons Attribution 4.0 International License (http://creativecommons. org/licenses/by/4.0/), which permits use, sharing, adaptation, distribution and reproduction in any medium or format, as long as you give appropriate credit to the original author(s) and the source, provide a link to the Creative Commons license and indicate if changes were made.

The images or other third party material in this book are included in the book's Creative Commons license, unless indicated otherwise in a credit line to the material. If material is not included in the book's Creative Commons license and your intended use is not permitted by statutory regulation or exceeds the permitted use, you will need to obtain permission directly from the copyright holder. 